\title{
Model order reduction in thermoacoustic stability analysis
}

\author{
Max Meindl*, Maria Cruz Varona*, \\ Alessandro Castagnotto*, Felix Thomann*, \\ Wolfgang Polifke*, Boris Lohmann* \\ * Technical University of Munich, Germany \\ (e-mail: meindl@tfd.mw.tum.de)
}

\begin{abstract}
Keywords: Model order reduction, state-space models, thermoacoustics, stability analysis,
\end{abstract} linear coupled systems.

\section{INTRODUCTION}

Modern gas-turbines are typically operated under lean, premixed combustion conditions due to emission restrictions. In this operating range, the flame is susceptible to acoustic perturbations. Once perturbed, the heat-release fluctuates, which acts as a monopole sound-source. Due to interaction with the compressor, the turbine and the combustor housing, these acoustic waves can be reflected back to the flame, which establishes a feedback cycle. When constructive interference occurs, the amplitudes of this mechanism can grow and a thermoacoustic instability develops that might limit the operating range, increase material wear and even cause fatal damage.

In order to predict and avoid these instabilities, linear time-invariant (LTI) models consisting of an acoustic part (AC) for the wave propagation and an acoustic-flame interaction model are often used. Due to the complex geometries found in gas-turbines, the models for the acoustic propagation, e.g. based on the finite element method, can become very large. In this contribution, a pilot study is conducted in which model order reduction (MOR) techniques are applied to a simple acoustic model. After the reduction, a flame transfer function (FTF) is coupled to the reduced acoustic system in order to account for the acoustic-flame interaction. This a posteriori coupling allows to change the FTF for parameter studies with only one MOR of the acoustic system. The linear thermoacoustic stability analysis is conducted on both, the full- (FOM) and the reduced-order model (ROM), by computing the eigenvalues of the coupled systems. The suitability of three MOR algorithms - modal reduction, truncated balanced realization and the iterative rational Krylov algorithm is investigated based on the stability prediction of the reduced models.

\section{THERMOACOUSTIC MODEL}

In this study, the thermoacoustic stability of a swirl burner is investigated. The model for the acoustic wave propagation is based on the $1 \mathrm{D}$ thermoacoustic network

\footnotetext{
* The work related to this contribution is supported by the German Research Foundation (DFG), Grant LO408/19-1.
}

modeling tool $t a X^{1}$, while the acoustic-flame interaction is governed by an FTF identified from a large eddy simulation of the burner. The state-space representation is used for both parts of the model, which facilitates easy coupling and robust computations.

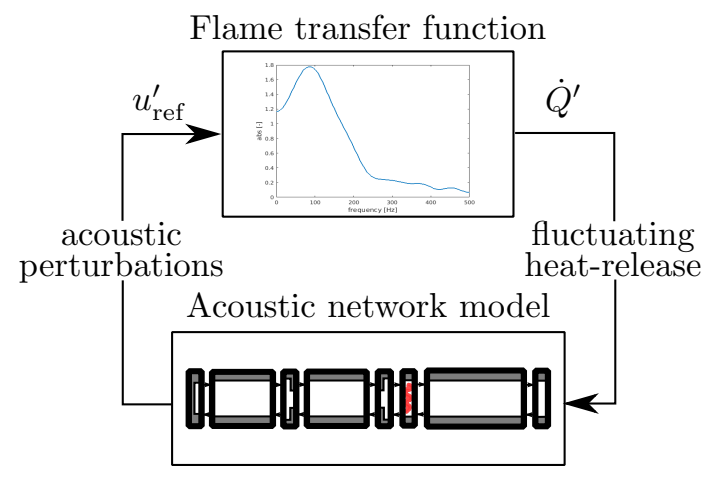

Fig. 1. Coupling between the acoustic and the flameresponse model.

\subsection{Acoustic network model}

Within the scope of this pilot study, a low order, 1D network model is used for the propagation of the acoustic waves. Although the size of this model is rather small 78 degrees of freedom - the basic principles learned from MOR should be applicable to larger models as well.

The network model consists of simple elements, for which the analytical solution of the wave propagation is known, e.g. a duct with constant cross-section or an area jump. By matching the wave amplitudes at the interfaces of these elements, a network that represents the whole burner can be established (see Fig. 1). In order to provide an interface to the FTF, inputs and outputs of the acoustic state-space model have to be chosen. Due to the velocity-sensitive premixed flame, the acoustic perturbation in axial flow velocity at a reference position $u_{\text {ref }}^{\prime}$ is chosen as an output. To account for the fluctuating heat-release $\dot{Q}^{\prime}$ of the flame, an input is provided which translates these fluctuations to acoustic waves.

1 The code for the software package taX can be found at http: //gitlab.lrz.de/tfd/tax and its documentation at http://tax. wiki.tum.de. 


\subsection{Flame transfer function}

The flame transfer function has a low-pass filter behavior and relates axial velocity perturbations to heat-release fluctuations. In state-space representation, the input of the FTF is $u_{\text {ref }}^{\prime}$ and the output is $\dot{Q}^{\prime}$. This allows for coupling between the FTF and the acoustic network model. Through the coupling, a monolithic state-space model is retrieved which incorporates the whole thermoacoustic dynamics.

\section{MODEL ORDER REDUCTION OF THE ACOUSTIC SUBSYSTEM}

Our pursued workflow consists of first reducing the acoustic part of the thermoacoustic model before coupling the acoustic ROM to the FTF. Another approach could be to reduce the coupled system $(\mathrm{AC}+\mathrm{FTF})$ as a whole. The former technique, however, is more advantageous, since it allows to perform parameter studies of the FTF without having to reduce the whole system after each parameter variation. To reduce the acoustic model, three linear MOR techniques from the $s s s M O R$ toolbox ${ }^{2}$ are applied (Castagnotto et al. (2017)). These techniques employ a projection of the FOM onto a respective subspace to obtain a corresponding ROM (Antoulas (2005)).

We compute the projection subspaces with different reduction methods, namely modal reduction (Davison (1966)), truncated balanced realization (Moore (1981)) and the iterative rational Krylov algorithm (Gugercin et al. (2008)). Note that modal reduction - i.e. the preservation of some eigenmodes - is state of the art in the reduction of acoustic models, since these can be usually extracted directly from FE solvers.

\section{COUPLING THE REDUCED ACOUSTIC SYSTEM WITH THE FLAME TRANSFER FUNCTION}

After the model order reduction, the reduced acoustic network model is coupled to the FTF. The eigenvalues of this reduced coupled model are compared to the eigenvalues of the full coupled model (full acoustic network model connected to FTF). The complexity of this task lies in the fact, that a good approximation of the eigenmodes of the acoustic subsystem will not guarantee a good fit of the coupled, thermoacoustic modes between the FOM and the ROM. This is due to the fact that the FTF significantly changes the system dynamics. The investigated model exhibits a low-frequent intrinsic thermoacoustic (ITA) eigenmode which is marginally stable. This mode only occurs in the coupled system (Hoeijmakers et al. (2014); Emmert et al. (2016)). Due to its prime importance for thermoacoustic stability, the quality of the ROM will be judged based on the approximation of this mode.

A robust and efficient MOR algorithm is desired, which produces a ROM that converges to the correct thermoacoustic eigenmodes, when coupled to the FTF. This study investigates the convergence behavior of the three before mentioned methods with respect to increasing ROM order.

\footnotetext{
2 sss and sssMOR are open-source MATLAB toolboxes available under www.rt.mw.tum.de/?morlab or in GitHub under https:// github.com/MORLab
}

It is known that the acoustic feedback mechanism between the fluctuating heat-release and the velocity perturbations at the reference position is of major importance for the intrinsic eigenmode. It is therefore expected that the model reduction algorithms focusing on exactly this transfer behavior from $\dot{Q}^{\prime}$ to $u_{\text {ref }}^{\prime}$ (TBR and IRKA) will yield better results than the modal MOR (cf. Fig. 2). This and further aspects will be discussed at the conference.

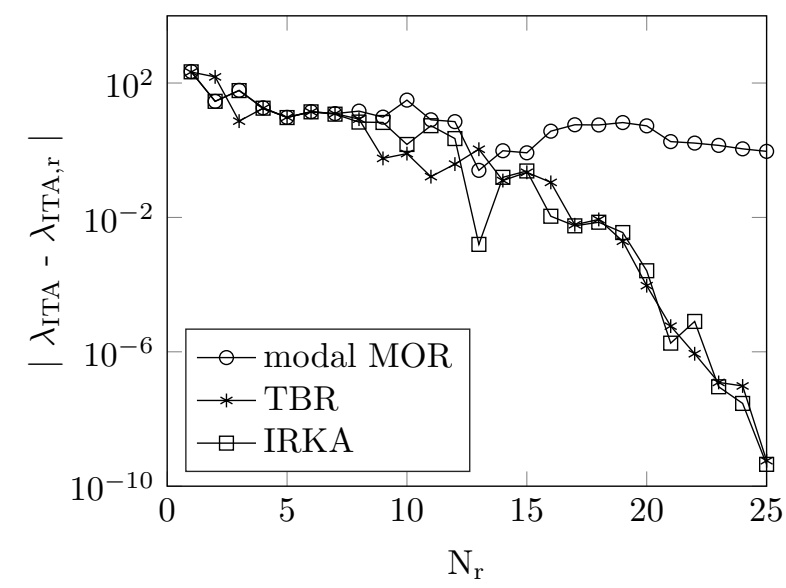

Fig. 2. Error between the ITA eigenfrequency of the FOM $\left(\lambda_{\text {ITA }}\right)$ and the ROMs $\left(\lambda_{\text {ITA,r }}\right)$ with the three different reduction algorithms depending on the order $N_{r}$ of the reduced acoustic system.

\section{OUTLOOK: LARGE-SCALE ACOUSTIC SYSTEMS}

Based on the experience gained from the small-scale network model case, suitable ROM techniques need to be developed for large-scale (aero-)acoustic models with up to $10^{6}$ degrees of freedom. Major challenges are the $3 \mathrm{D}$ effects and hydrodynamic-acoustic interactions, which are not included in the network model.

\section{REFERENCES}

Antoulas, A.C. (2005). Approximation of Large-Scale Dynamical Systems. SIAM. doi:10.1137/1.9780898718713.

Castagnotto, A., Cruz Varona, M., Jeschek, L., and Lohmann, B. (2017). sss \& sssmor: Analysis and Reduction of Large-Scale Dynamic Systems in MATLAB. at-Automatisierungstechnik, 65(2).

Davison, E. (1966). A method for simplifying linear dynamic systems. IEEE TAC, 11(1), 93-101.

Emmert, T., Bomberg, S., Jaensch, S., and Polifke, W. (2016). Acoustic and Intrinsic Thermoacoustic Modes of a Premixed Combustor. In 36th Int'l Symposium on Combustion. Combustion Institute, Seoul, Korea. doi: 10.1016/j.proci.2016.08.002.

Gugercin, S., Antoulas, A.C., and Beattie, C. (2008). $\mathcal{H}_{2}$ Model Reduction for Large-Scale Linear Dynamical Systems. SIAM Journal on Matrix Analysis and Applications, 30(2), 609-638. doi:10.1137/060666123.

Hoeijmakers, M., Kornilov, V., Lopez Arteaga, I., de Goey, P., and Nijmeijer, H. (2014). Intrinsic Instability of Flame-Acoustic Coupling. Combustion and Flame, 161(11), 2860-2867.

Moore, B.C. (1981). Principal component analysis in linear systems: controllability, observability and model reduction. IEEE TAC, 26, 17-32. 